\title{
ARTICLE OPEN \\ Passivation behaviour of aluminium current collector in ionic liquid alkyl carbonate (hybrid) electrolytes
}

\author{
Sowmiya Theivaprakasam ${ }^{1,2,3}$, Gaetan Girard $\mathbb{D}^{4}$, Patrick Howlett $\mathbb{D}^{4}$, Maria Forsyth ${ }^{4}$, Sagar Mitra ${ }^{2}$ and Douglas MacFarlane ${ }^{3}$
}

The compatibility of current collectors with the electrolyte plays a major role in the overall performance of lithium batteries, critical to obtain high storage capacity as well as excellent capacity retention. In lithium-ion batteries, in particular with cathodes that operate at high voltage such as lithium nickel cobalt manganese oxide, the cathodic current collector is aluminium and it is subjected to high oxidation potentials $\left(>4 \mathrm{~V}\right.$ vs. $\left.\mathrm{Li} / \mathrm{Li}^{+}\right)$. As a result, the composition of the electrolyte needs to be carefully designed in order to stabilise the battery performance as well as to protect the current collectors against corrosion. This study examines the role of a hybrid electrolyte composed of an ionic liquid ( $N$-methyl- $N$-propyl pyrrolidinium bis(trifluoromethanesulfonyl)imide or $N$ methyl- $N$-propyl pyrrolidinium bis(fluorosulfonyl)imide) and a conventional electrolyte mixture (LiPF 6 salt and alkyl carbonate solvents) with correlation to their electrochemical behaviour and corrosion inhibition efficiency. The hybrid electrolyte was tested against battery grade aluminium current collectors electrochemically in a three-electrode cell configuration and the treated aluminium surface was characterised by SEM/EDXS, optical profilometry, FTIR, and XPS analysis. Based on the experimental results, the hybrid electrolytes allow an effective and improved passivation of aluminium and lower the extent of aluminium dissolution in comparison with the conventional lithium battery electrolytes and the neat ionic liquids at high anodic potentials $\left(4.7 \mathrm{~V} \mathrm{vs}\right.$. Li/Li ${ }^{+}$). The mechanism of passivation behaviour is also further investigated. These observations provide a potential direction for developing improved hybrid electrolytes, based on ionic liquids, for higher energy density devices.

npj Materials Degradation (2018)2:13; doi:10.1038/s41529-018-0033-6

\section{INTRODUCTION}

In a lithium-ion battery, the flow of lithium ions between the electrodes happens along with the electron transfer in the external circuit. The electron transfer is achieved with the help of thin metal foils which act as current collectors, which are coated with the active electrode materials in the battery. ${ }^{1-3}$ Since the primary role of the current collectors is electron conduction, high electronic conductivity as well as high stability in that electrochemical environment are of crucial importance. ${ }^{1-4}$ Though thin foils of nickel, copper, platinum, zinc, titanium, and other similar metals have been studied as current collectors, aluminium (Al) is the most preferred cathodic current collector in lithium-ion batteries due to its ability to form a passive film, making the electrolyte/Al interface stable even at potentials $>4 \mathrm{~V}$ vs. $\mathrm{Li} / \mathrm{Li}^{+}$. Al also offers light weight, low cost, good adhesion to the active materials, and good electronic conductivity. ${ }^{3,4}$ The current use of lithium-ion batteries in electric vehicles requires safe, high energy density batteries., ${ }^{5,6}$ One area which is very widely studied for high energy lithium batteries is the development of cathodes that operate at high voltage. ${ }^{7,8}$ Improved stability of the aluminium current collectors is also important to realise the improvement in performance offered by these high potential cathodes., ${ }^{3,9-11}$ In addition, several reports ${ }^{12-14}$ in the literature also claim that the electrolyte composition, especially the nature of the salt, plays a major role in the corrosion of aluminium current collectors; some other reports claim that the solvents play a role. ${ }^{15,16}$ In most commercially used electrolytes, lithium hexafluoro phosphate $\left(\mathrm{LiPF}_{6}\right)$ is the preferred salt due to the passivation mechanism on aluminium, despite its thermal instability. ${ }^{11,13,17}$ Salts with better thermal stability such as the lithium bis(trifluoromethanesulfonyl) imide (LiTFSI) are not preferred because they tend to corrode aluminium current collectors. ${ }^{13,18}$ Although the state of the art electrolytes with $\mathrm{LiPF}_{6}$ salts work well under present day operating potentials, they still cause corrosion of current collectors at the potentials where high potential cathodes are expected to operate. $^{19}$

Recent studies examined the use of ionic liquids as electrolyte components in lithium batteries and selected ionic liquids were found to possess wide electrochemical stability, improved safety ${ }^{15,17,20-22}$ as well as corrosion inhibition properties. ${ }^{23-26}$ It is interesting to note that the well-known corrosive nature of the LiTFSI salt is decreased when ionic liquids were added to the electrolyte composition. ${ }^{17,27,28}$ In addition to the corrosion inhibition, the so-called passive films formed on the substrate in the presence of ionic liquid electrolytes show better thermal stability. ${ }^{27}$ On the other hand, in spite of the corrosive nature of the bis(fluorosulfonyl) imide anion [FSI] at high oxidation potentials $\left(>4.0 \mathrm{~V}\right.$ vs. $\mathrm{Li} / \mathrm{Li}^{+}$), Cho et al. demonstrated that the addition of $\mathrm{LiPF}_{6}$ to $\mathrm{N}$-methyl- $N$-propyl pyrrolidinium bis(fluorosulfonyl)imide $\left[\mathrm{C}_{3} \mathrm{mpyr}\right][\mathrm{FSI}]$ reduced pitting corrosion. ${ }^{29}$

Despite their remarkable thermal stability, the use of ionic liquid mixtures as electrolytes in lithium batteries is limited by their low ionic conductivity at room temperatures as well as issues in solid

\footnotetext{
${ }^{1}$ IITB-Monash Research Academy, Powai, Mumbai 400076, India; ${ }^{2}$ Department of Energy Science and Engineering, Indian Institute of Technology Bombay (IITB), Mumbai 400076,

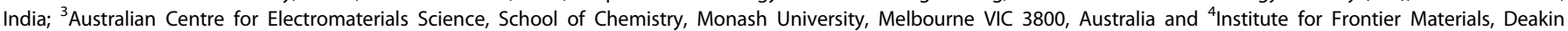
University, Burwood VIC 3125, Australia

Correspondence: Maria Forsyth (maria.forsyth@deakin.edu.au) or Sagar Mitra (sagar.mitra@iitb.ac.in) or Douglas MacFarlane (douglas.macfarlane@monash.edu)
}

Received: 24 October 2017 Revised: 30 January 2018 Accepted: 11 February 2018

Published online: 29 March 2018 
electrolyte interphase formation. ${ }^{30-32}$ To overcome these disadvantages, researchers have introduced the concept of hybrid electrolytes, where an organic solvent is added to the ionic liquid electrolytes so as to increase the room temperature ionic conductivity as well as to get better interfacial behaviour. ${ }^{15,20,33-36}$

In this work, we have tested hybrid electrolytes composed of $40 \mathrm{wt} \%$ ionic liquid in a conventional electrolyte system to understand the role of the ionic liquid in the passivation mechanism of aluminium foil current collector. The proportion of $40 \mathrm{wt} \%$ ionic liquid was chosen as a compromise between improved safety and low viscosity for these electrolytes at room temperatures..$^{20,34,37}$ The most commonly studied ionic liquids in lithium batteries ${ }^{20,33,38,39}$ such as $\left[\mathrm{C}_{3} \mathrm{mpyr}\right][\mathrm{TFSI}]$ and $\left[\mathrm{C}_{3} \mathrm{mpyr}\right][\mathrm{FSI}]$ were used in hybrid combinations with the conventional electrolyte mixture. Electrochemical tafel studies were carried out to understand the corrosion kinetics in these hybrid electrolytes. Surface techniques such as scanning electron microscopy (SEM), energy dispersive x-ray spectroscopy (EDXS) and optical profilometry were employed to understand the extent of aluminium passivation. Furthermore, an understanding of the passive film composition was obtained using Fourier transform infrared spectroscopy (ATR-FTIR) and x-ray photoelectron spectroscopy (XPS) studies. The current findings support the synergistic role of hybrid electrolytes in bringing better surface passivation on aluminium which may improve the performance of lithium batteries, especially for high energy density cathode systems.

\section{RESULTS}

Electrochemical measurements

Potentiodynamic measurements were carried out to understand the corrosion kinetics in hybrid electrolytes, conventional electrolytes and neat ionic liquids. The potentiodynamic polarisation curves are illustrated in Fig. 1. The corrosion current densities were lower in the electrolytes containing ionic liquids compared with the conventional electrolytes. In hybrid electrolytes, the corrosion potential as well as corrosion current density initially follows the same trend as that seen for the neat ionic liquids; however, at more positive potentials, the presence of $\mathrm{LiPF}_{6}$ in the system creates passivation. This passivation phenomenon can be seen by the flat regions in the curves for both conventional and hybrid electrolytes. This clearly shows that the hybrid electrolytes can help decrease corrosion of aluminium current collectors.

Further insights into this corrosion phenomenon were obtained by performing cyclic voltammetry on the aluminium current collectors with the electrolytes under study. This aimed to (i) verify the passive film formation, (ii) understand whether the formation is a once-off process or whether the film has self-healing properties and (iii) establish the mechanism of passive film formation and its composition.

We initially carried out CV measurements over a potential range of $2.7-4.7 \mathrm{~V}$ vs. $\mathrm{Li} / \mathrm{Li}^{+}$as shown in Fig. 2 . The cycle was repeated twice at a rate of $0.1 \mathrm{mV} / \mathrm{s}$ to understand the passive film formation. Quasi-flat profiles in the CV during the second and third cycles were found with IL-based electrolytes, as seen in Fig. 2. In contrast, a significant constant increase of current density with respect to the potential sweep was observed in the neat LP40 electrolyte.

Following the CV measurements, chronoamperometry was carried out, in which the samples were subjected to a potential of $4.7 \mathrm{~V} \mathrm{vs.} \mathrm{Li} / \mathrm{Li}^{+}$for three hours to further examine the strength of the passive film. The flat lines (i.e., reflecting a constant current) indicate that the passive film is further impeding corrosion reactions as shown in Figure S1. The current versus time profiles confirm that the film formed in the LP40 electrolyte is not sufficiently passive so as to prevent the surface from corrosion, as seen from the steady increase of current in Fig. 2a. On the other hand, the surface film that develops in neat ionic liquids as well as the hybrid electrolytes prevented further corrosion of the $\mathrm{Al}$ current collector as seen from the very low current densities measured in the second and third cycles (Fig. 2b-e) and in the chronoamperometry experiments. The slight increase in current density values measured after 1.5 hours in the HE 2 electrolyte (Fig S1) may suggest a less protective or damaged passive film formed in this case. Further surface characterisations were conducted on these samples to understand the formation mechanism and composition of the passive film.

\section{Surface analyses}

The surfaces of the electrochemically treated aluminium current collectors were analysed using SEM, EDXS, and optical profilometry whereas the surface composition was further analysed by FTIR and XPS analysis.

SEM \& EDXS studies. Figure 3 shows SEM images of aluminium current collectors after anodising in the different electrolytes. Close observation of SEM images indicated the formation of a layer on the surface in all cases, showing passivation by chemical reaction.

The SEM micrograph of Fig. 3a shows the surface morphology of the pristine surface before any test. The treated surface in the organic carbonate solvent (LP40) appears significantly roughened, Fig. 3 b. Closer observation reveals the formation of a thick and non-uniform passive layer in the sample exposed to the LP40 electrolyte. EDXS analysis confirmed the presence of a relatively thick layer on the Al surface, with a chemical composition
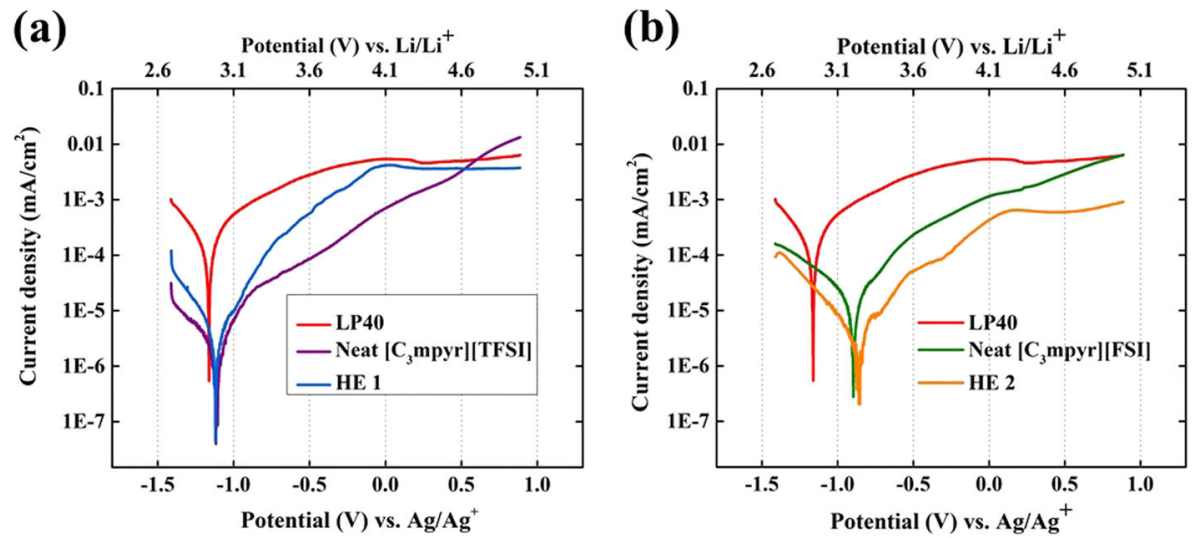

Fig. 1 Tafel plots of aluminium foils in (a) $\left[\mathrm{C}_{3} \mathrm{mpyr}\right][\mathrm{TFSI}]$ ionic liquid and $\mathrm{HE} 1$ in comparison with LP40 and (b) [C $\left.\mathrm{C}_{3} \mathrm{mpyr}\right][\mathrm{FSI}]$ ionic liquid and HE 2 in comparison with LP40 
(a)

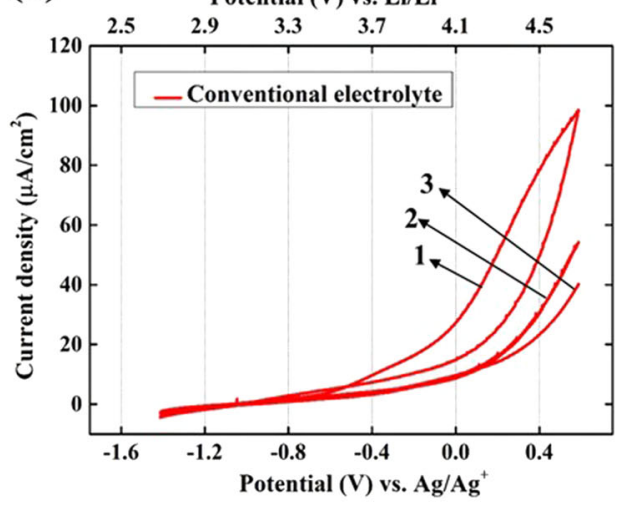

(c)

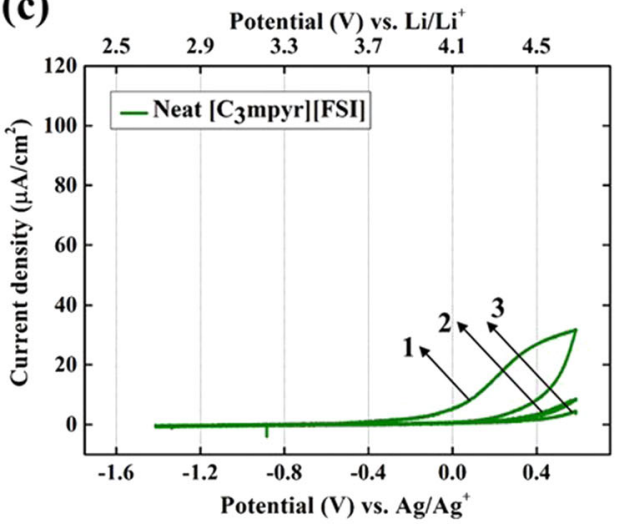

(b)

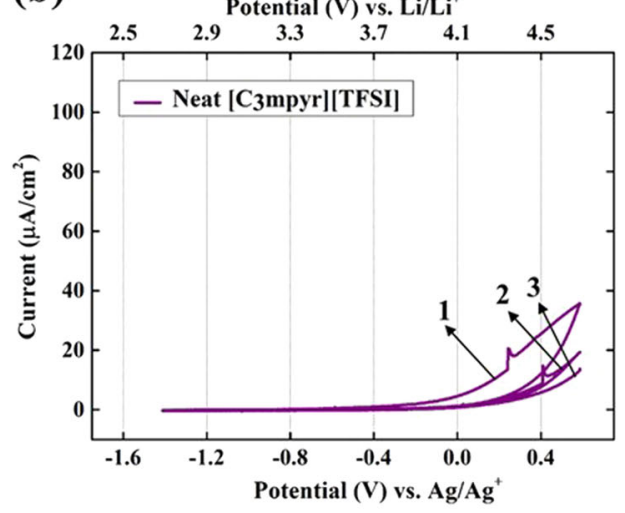

(d)

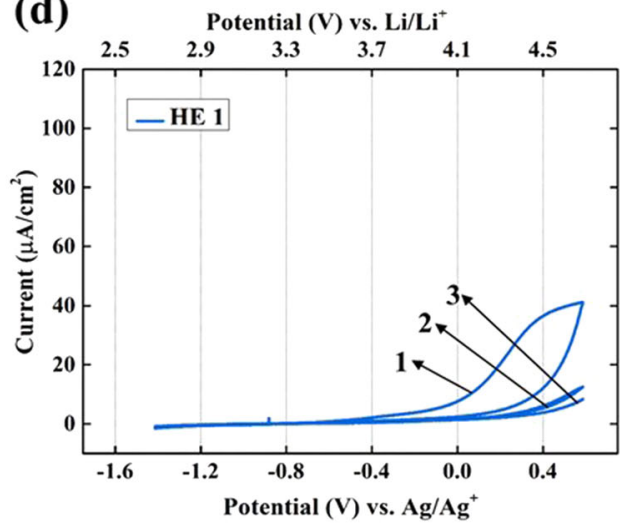

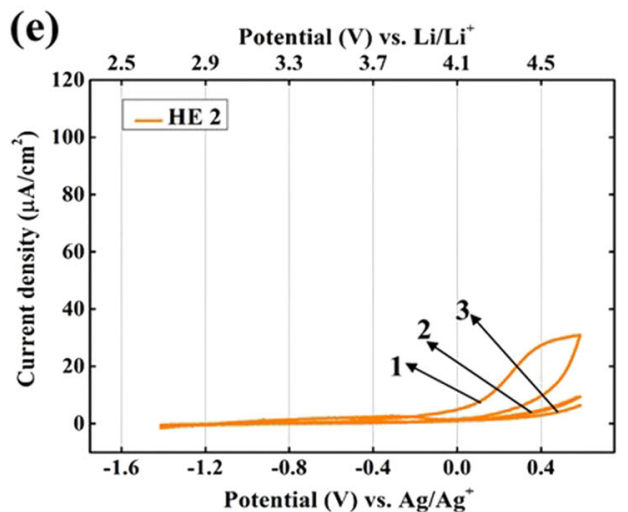

Fig. 2 Cyclic voltammograms of aluminium foils in (a) LP40, (b) [ $\left.\mathrm{C}_{3} \mathrm{mpyr}\right]\left[\mathrm{TFSI}\right.$, (c) [C $\left.\mathrm{C}_{3} \mathrm{mpyr}\right][\mathrm{FSI}]$, (d) HE 1, and (e) HE 2 at a scan rate of $0.1 \mathrm{mV} /$ $\mathrm{s}$ in the potential window of $2.7-4.7 \mathrm{~V} \mathrm{vs}$. $\mathrm{Li}^{\prime} / \mathrm{Li}^{+}$. The numbers indicated by arrows refer to the cycle number of the cyclic voltammogram trace

dominated by phosphorus, carbon, fluorine and oxygen, as shown in Figure S2. The surface morphologies in Fig. 3c, d show evidence that the [TFSI] ${ }^{-}$anion helps protect the aluminium surface through formation of protective layer stable against the anodising process, resulting in a smoother surface. Smaller amounts of the elements associated with the [TFSI] ${ }^{-}$anion were detected by EDXS (Figures S3 and S4), although these need to be further confirmed by XPS analysis.

The surface morphology in the case of the neat [FSI] sample is very different to that of the neat [TFSI] sample (Fig. 3c, e). The [FSI] treated sample has a very uneven surface, suggesting a heterogeneous passivation layer on the Al surface (Figure S5). The main difference seen in the SEM analysis of the surface exposed to the hybrid electrolyte (HE 1) is the presence of a smoother deposit when the ionic liquid with the [TFSI] anion is mixed with LP40, Fig. 3e (HE 1 sample). The cracks visible on the surface are likely to be a result of the vacuum in the SEM chamber. Larger quantities of elements such as $\mathrm{O}, \mathrm{F}$, and $\mathrm{S}$ associated with the anion in the case of the HE 2 surface were confirmed by EDXS analysis (Figure S6).

Optical profilometric study. To obtain quantitative information about the surface roughness, the samples treated in neat ionic liquids, HE 1, and HE 2 were examined by optical profilometry. The 3D images are given in Fig. 4.

After exposure to the neat ILs (Fig. 4a, b, respectively for the neat [TFSI] and neat [FSI] samples), there were rough, distributed deposits on the surface, and the roughness was significantly more pronounced in the case of the neat [FSI] sample. The neat [TFSI], HE 1 and HE 2 samples showed similar average roughness. However, 


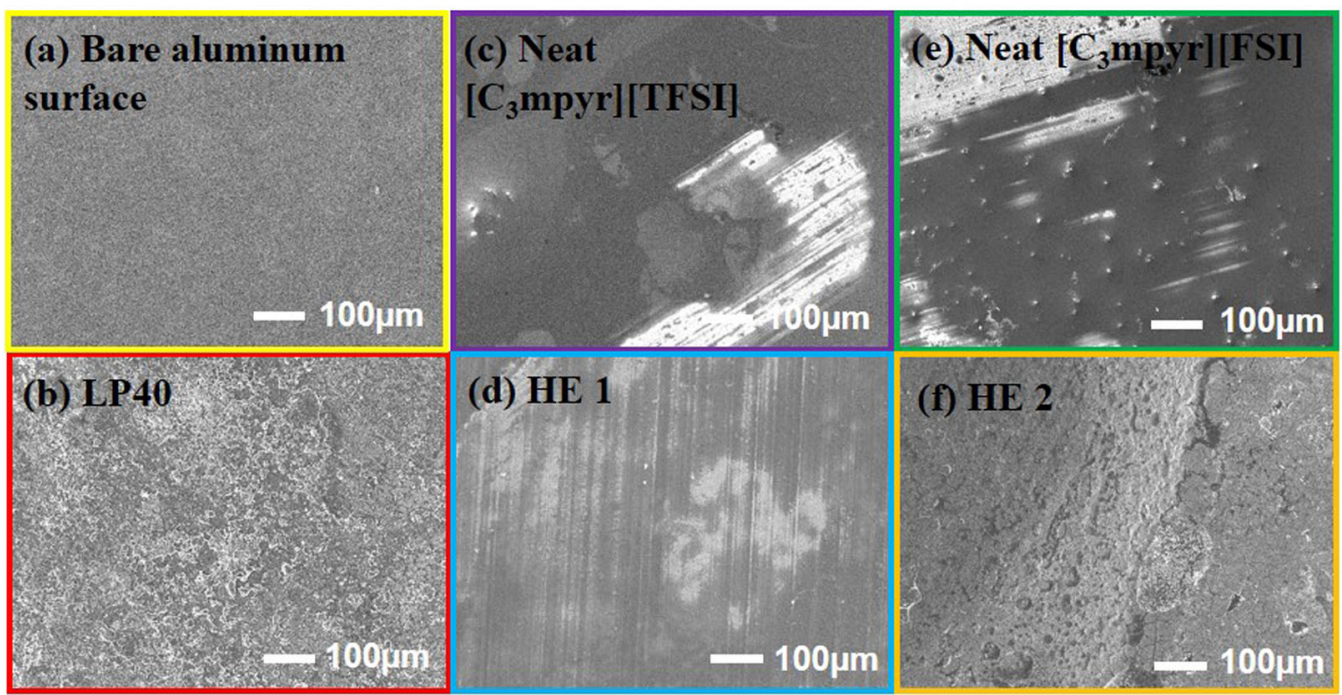

Fig. $3 \mathrm{SEM}$ images $(\mathrm{EHT}=10 \mathrm{kV}$ ) of aluminium foil (a) bare aluminium surface and after electrochemistry measurements in (b) LP40, (c) $\left[\mathrm{C}_{3} \mathrm{mpyr}\right][\mathrm{TFSI}],(\mathbf{d})\left[\mathrm{C}_{3} \mathrm{mpyr}\right][\mathrm{FSI}],(\mathbf{e}) \mathrm{HE} 1$, and (f) HE 2
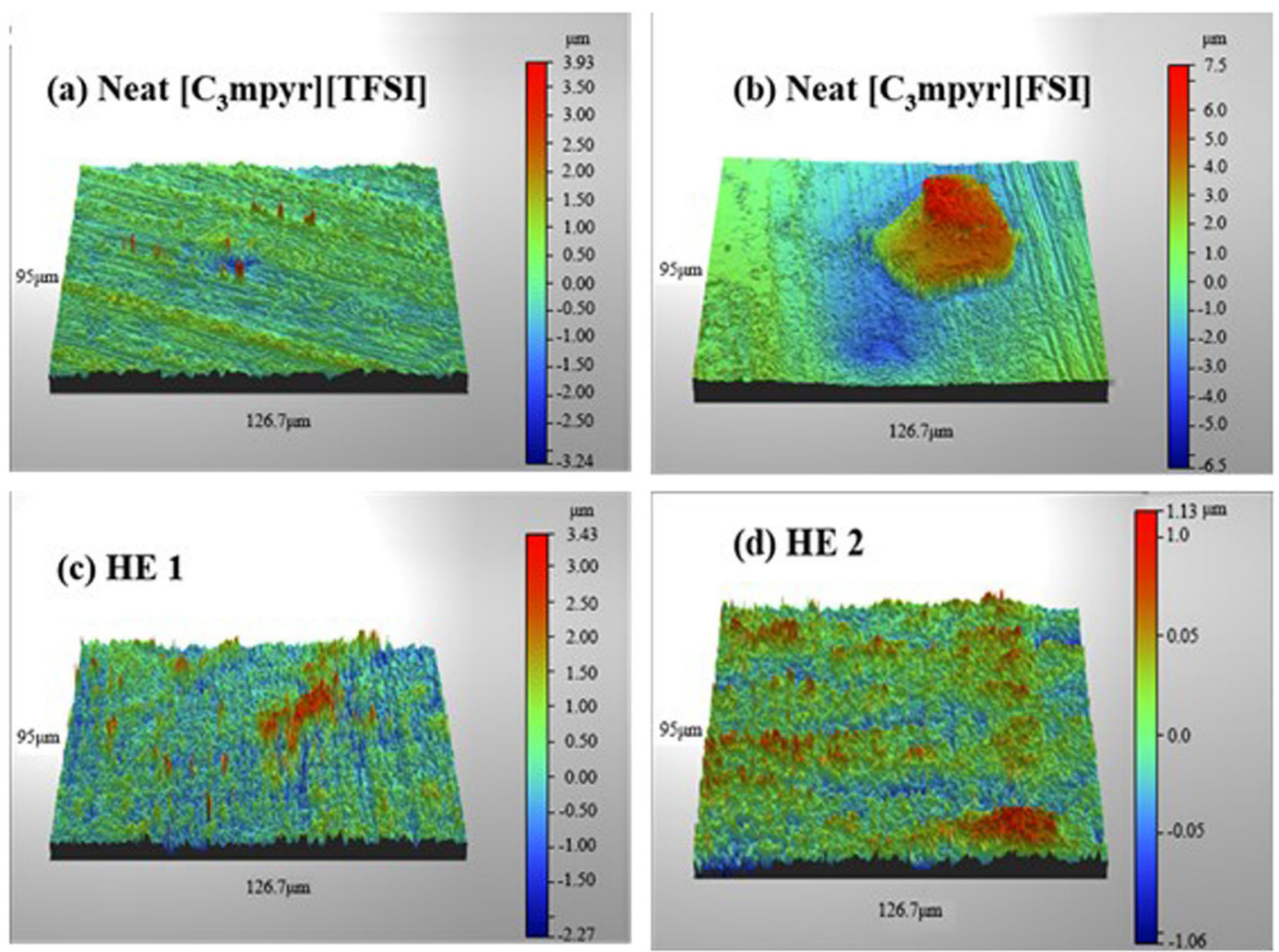

Fig. 4 Profilometric 3D images collected for aluminium surfaces after anodising in (a) $\left[\mathrm{C}_{3} \mathrm{mpyr}\right]\left[\mathrm{TFSI}\right.$, (b) $\left[\mathrm{C}_{3} \mathrm{mpyr}\right][\mathrm{FSI}]$, (c) $\mathrm{HE} 1$, and (d) $\mathrm{HE} 2$ obtained by optical profilometry

evenly distributed layers are more pronounced in $\mathrm{HE} 1$ and $\mathrm{HE}$ 2 samples, Fig. 4c, d, respectively for HE 1 and HE 2. This observation is confirmed by a more stable relative height and smaller errors in roughness average, as illustrated in Figure S7. These parameters reflect the homogeneity and distribution of the layer across the surface. These images are consistent with those from SEM (Fig. 3). Here it must be noted that the colour legend on the right side of the images in Fig. 4 is different for each sample, therefore attention should be drawn to the legend before making comparisons.
The observations from the profilometric study agree with previous SEM analysis. The results provide evidence of a more uniform protective layer formed with the [TFSI]-based hybrid system. The average roughness was measured on multiple spots and small deviations obtained in the case of hybrid samples reflect the uniformity of the surface as shown in Table S7. This could explain the electrochemical results (CV) described above. In the case of neat [TFSI] and neat [FSI], the surface was not flat (this observation is confirmed by a relative height variation of $\pm 10 \mu \mathrm{m}$, 
Neat $\left[\mathrm{C}_{3} \mathrm{mpyr}\right][\mathrm{TFSI}]$
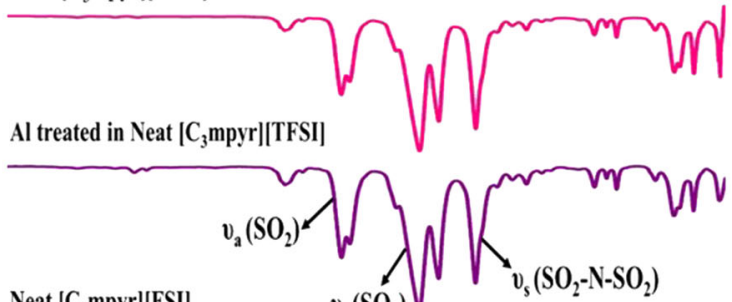

Neat $\left[\mathrm{C}_{3}\right.$ mpyr $][\mathrm{FSI}]$ $v_{s}\left(\mathrm{SO}_{2}\right)$

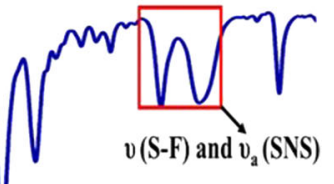

Al treated in Neat $\left[\mathrm{C}_{3} \mathrm{mpyr} \mid[\mathrm{FSI}]\right.$
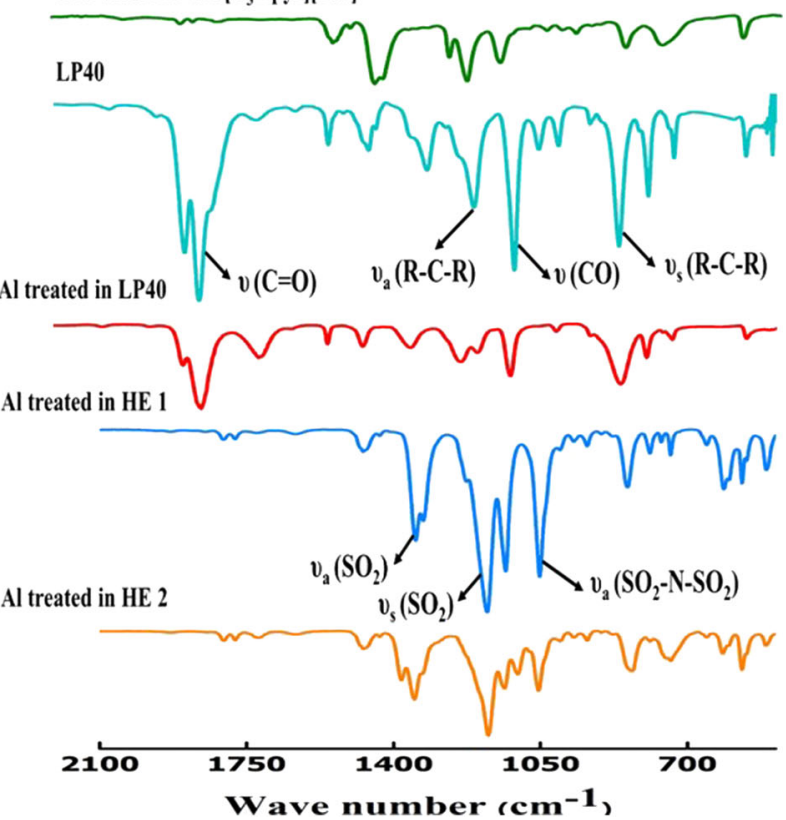

Fig. $5 \mathrm{FTIR}$ spectra of neat $\left[\mathrm{C}_{3} \mathrm{mpyr}\right][\mathrm{TFSI}],\left[\mathrm{C}_{3} \mathrm{mpyr}\right][\mathrm{FSI}]$ as well as the aluminum foils treated in $\left[\mathrm{C}_{3}\right.$ mpyr][TFSI], [ $\left.\mathrm{C}_{3} \mathrm{mpyr}\right][\mathrm{FSI}], \mathrm{HE} 1$ and HE 2

Figure S7) whereas a smoother surface was confirmed in the case of the HE 1 sample.

FTIR analysis. FTIR spectra of the electrochemically treated aluminium foils are shown in Fig. 5. In the case of hybrid electrolytes, the strong peaks corresponding to $\mathrm{SO}_{2}$ asymmetrical and symmetrical stretching were found at 1177 and $1348 \mathrm{~cm}^{-1}$ for the sample in $\mathrm{HE} 1$ and 1174 and $1381 \mathrm{~cm}^{-1}$ for $\mathrm{HE} \mathrm{2,} \mathrm{respectively.}$ This indicates that the $\mathrm{SO}_{2}$ portion of the anion has remained intact and present on or very near the surface ${ }^{40-42}$ (taking into account, the penetration depth of the IR beam). The $\mathrm{SO}_{2}$ group may belong to multiple species on the aluminium surface but these FTIR spectra do not enable any conclusion to be made about the exact chemical species present. There does not seem to be a peak that could be assigned to the S-F stretch. ${ }^{41}$ The strong peaks corresponding to $\mathrm{S}-\mathrm{N}$ stretch are clearly present in the aluminium electrode spectrum at $\sim 740$ and $843 \mathrm{~cm}^{-1}$ in HE 1 and $\sim 740$ and $832 \mathrm{~cm}^{-1}$ for HE 2, respectively. ${ }^{41,43-45}$ All $\mathrm{C}-\mathrm{H}$-based vibrations that do not arise from the DMC reaction residue must be due to the $\left[\mathrm{C}_{3}\right.$ mpyr $]$ cation. There are clear peaks associated with the $\mathrm{CH}_{3}$ stretch at 2888, 2947, and $2980 \mathrm{~cm}^{-1}$ in the spectrum of the sample in HE 2, which precisely match the peak positions ${ }^{41,42,45,46}$ found in the spectra of the neat $\left[C_{3}\right.$ mpyr $][$ TFSI $]$ and $\left[C_{3}\right.$ mpyr $][F S I]$ as shown in Fig. 5. This indicates that, at least in most cases, the alkyl chains are preserved and suggest the presence of the intact pyrrolidinium cation on the surface. During the anodising process, the Al surface becomes positively charged, so it is expected that migration of the anion towards Al will be promoted as aluminium is being dissolved into the solution $\left(\mathrm{Al} \rightarrow \mathrm{Al}^{3+}\right)$, whilst the cation should be repelled from the surface.

Indeed, literature reports based on the elemental composition of ionic liquid films on various reactive metals show that the ionic liquid anion greatly dominates the surface chemistry. ${ }^{47}$ However, other studies using ToF-SIMS revealed that both the cation and anion are present in the film although only the cation could be identified as a whole molecule. ${ }^{26}$ This confirms that the anion reactivity plays a dominant role in the passivation of aluminium current collectors whilst the cation is somehow entrapped within the passive surface film.

XPS analysis. XPS measurements-XPS high-resolution region spectra peak identities were determined from published values $^{48,49}$ and from reports of XPS studies of aluminium surfaces. ${ }^{50,51}$

The data obtained initially (before etching) are indicative of the outer surface. Figure 6 a presents the survey spectra obtained from the neat IL samples and the corresponding hybrid samples. Chemical compositions (atomic\%) are included in electronic supplementary information (ESI) (Table S2). Measurements clearly indicate that elements associated with the IL anions were present on the surface of the samples in significant quantities. Differences in chemical composition before and after etching are more pronounced in the case of neat ILs, suggesting that the surfaces treated in the hybrid solutions are more homogeneous. Larger amounts of $\mathrm{F}$, as expected, and interestingly larger amounts of Al, were detected in the sample surfaces treated in [TFSI] containing electrolytes (neat and HE 1) whereas more $C$ was detected in the [FSI] treated samples (neat and HE 2).

XPS etching measurements-Although the etching procedure is destructive and sample compositions must be analysed with care, it may be useful to reveal species likely to be present in the passivation layer, providing significant insight into the chemical nature of the film. High-resolution region spectra were acquired initially and after $5 \mathrm{~min}$ of etching. As the etching rate was estimated at $2 \AA . s^{-1}$, the experiment is estimated to have removed approximately $60 \mathrm{~nm}$ of material. The results obtained from the Al $2 \mathrm{p}$ and $\mathrm{F} 1 \mathrm{~s}$ for the four samples are summarised in Fig. 6b, c. Region spectra from $\mathrm{C} 1 \mathrm{~s}, \mathrm{~N} \mathrm{1s}, \mathrm{O} 1 \mathrm{~s}$, and $\mathrm{S} 2 \mathrm{p}$ are provided in ESI (Figures S8 to S11) along with peak assignments (Tables S3 and S4).

Considering the $F$ is spectra, peaks were identified for aluminium fluoride $\left(\mathrm{AlF}_{3}\right)$ at ca. $686.3 \mathrm{eV}$ and $-\mathrm{SO}_{2} \mathrm{~F}$ at ca. $688 \mathrm{eV}$ for all samples. There was an increase in the quantity of $\mathrm{AlF}_{3}$ in all samples except for the neat [FSI] treated sample. The results suggest that a layer of $\mathrm{AlF}_{3}$ is present close to the aluminium surface. Peaks associated with $-\mathrm{CF}_{3}$, a "fingerprint" of the [TFSI $\left.{ }^{-}\right]$ anion, were identified in the [TFSI] treated samples at $\mathrm{ca}$. 688.7-689 eV; similar amounts were detected in the neat [TFSI] treated sample before and after etching whereas the amount of $-\mathrm{CF}_{3}$ present in the hybrid HE 1 treated sample decreased drastically with etching. There are significant differences between the samples with the surface films formed from neat ILs or the HE 1 electrolyte containing mainly $-\mathrm{SO}_{2} \mathrm{~F}$ and $\mathrm{AlF}_{3}$, after a small amount of etching whereas a more steady variation in composition was exhibited by the HE 2 sample, suggesting that the Al film may be thicker or significant amounts of other species may be present on the Al surface in this latter case.

The $\mathrm{Al} 2 \mathrm{p}$ high-resolution region spectra displayed peaks corresponding to $\mathrm{Al}^{0}$ at $71-72 \mathrm{eV}$, aluminium oxide $\left(\mathrm{Al}_{2} \mathrm{O}_{3}\right)$ at ca. $74.6 \mathrm{eV}, \mathrm{Al}_{2} \mathrm{O}_{3}$ on $\mathrm{Al}$ foil at ca. $75.6 \mathrm{eV}$ and $\mathrm{AlF}_{3}$ at ca. 76.6 eV. The initial Al surface spectra indicated the presence of a considerable 

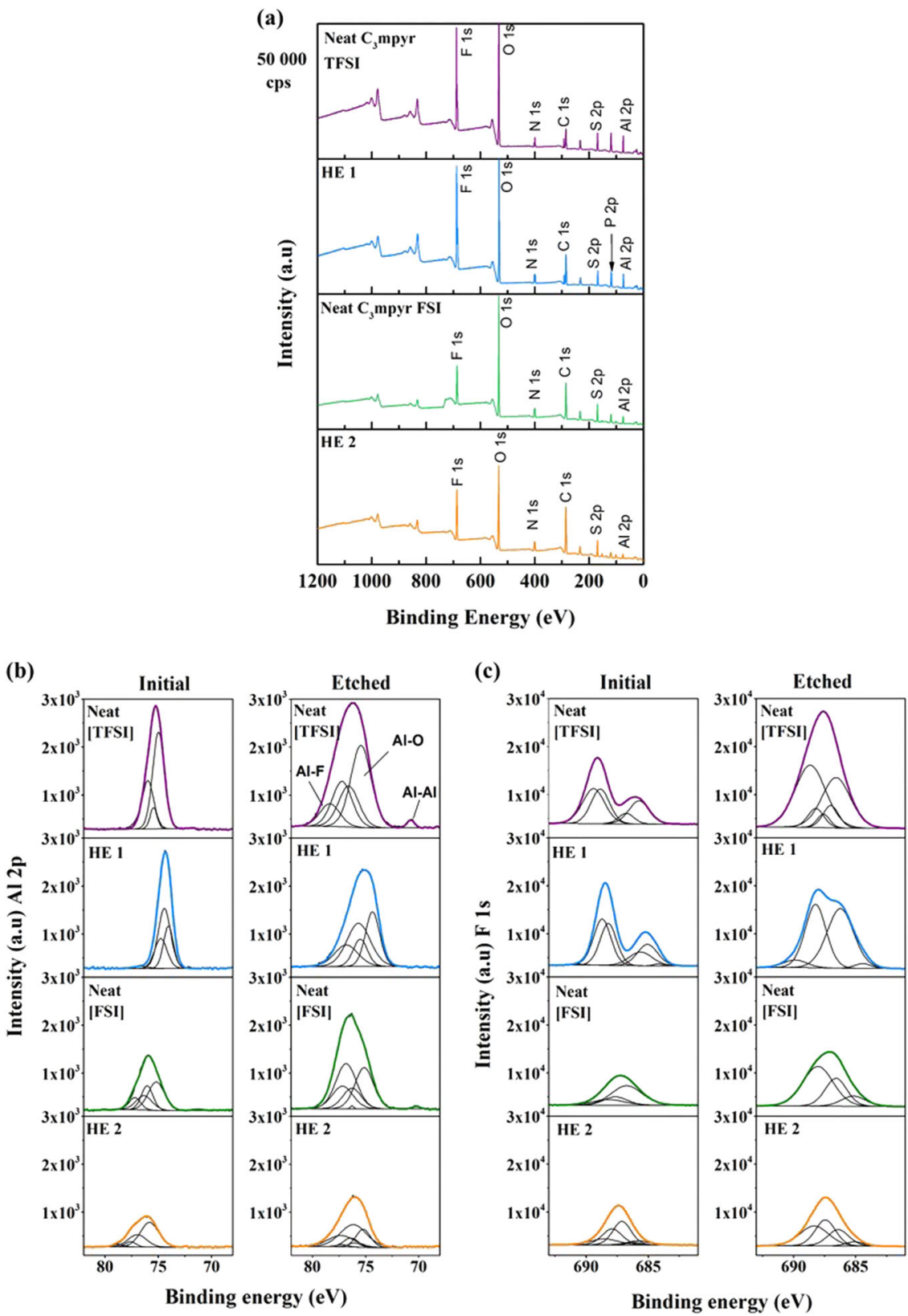

Fig. 6 a XPS survey scan and $\mathbf{b}$ high resolution Al $2 p$ and $F 1 s$ region spectra for the Al surfaces treated in Neat $\left[C_{3} m p y r\right][T F S I], H E ~ 1, ~ N e a t$ $\left[\mathrm{C}_{3} \mathrm{mpyr}\right][\mathrm{FSI}]$ and $\mathrm{HE} 2$ solutions before and after etching

quantity of Al oxide that did not persist well with etching, and was most likely present as a contaminant from sample handling. A substantial amount of $\mathrm{AlF}_{3}$ is indicated on the Al surface in the case of the FSI electrolytes (neat [FSI] and HE 2) and the quantity of $\mathrm{AlF}_{3}$ continued to increase with etching. $\mathrm{Al}^{\circ}$ associated with the Al substrate was only detected in the surfaces treated in neat ILs, suggesting the formation of thinner films, in agreement with the survey scan described previously and results obtained from other techniques. If the etching experiment is estimated to have removed $60 \mathrm{~nm}$ of material, the x-ray beam has not penetrated through the passivating film in the case of hybrid samples. After etching, a relatively complex mixture of aluminium species was identified in most samples and the response to the fitting parameters was satisfactory. Spin orbit splitting generates a doublet corresponding to one Al $2 \mathrm{p}$ species, the doublet separation should be of $1.15 \mathrm{eV}$ with a ratio of $2: 1{ }^{49}$
From the $\mathrm{C}$ 1s high-resolution region spectra, peaks associated with $\mathrm{C}-\mathrm{C} / \mathrm{C}-\mathrm{H}$ at $284.8 \mathrm{eV}, \mathrm{C}-\mathrm{N}$ at ca. $286.5 \mathrm{eV}$ and carbonate $\left(\mathrm{CO}_{3}{ }^{2-}\right)$ at ca. $290 \mathrm{eV}$ were identified.

Peaks detected in the $\mathrm{O} 1 \mathrm{~s}$ high-resolution region spectra were assigned to $\mathrm{CO}_{3}{ }^{2-}$, organic $\mathrm{C}-\mathrm{O}$ or $\mathrm{SO}_{2}{ }^{-}$at ca. $531.5 \mathrm{eV}, \mathrm{C}=\mathrm{O}$ at $533 \mathrm{eV}$. The $\mathrm{C} 1 \mathrm{~s}$ and $\mathrm{S} 2 \mathrm{p}$ spectra indicate that predominately $\mathrm{SO}_{2}$ was detected. The spectra show that the amount of $\mathrm{CO}_{3}{ }^{2-}$ and $\mathrm{SO}_{2}{ }^{-}$ decreased upon etching except for the neat [FSI] treated sample.

Peaks present in the $\mathrm{N}$ 1s high-resolution region spectra were associated with $\mathrm{N}^{+}$at $402-403 \mathrm{eV}$ and $\mathrm{N}^{-}$at $399-400 \mathrm{eV}$. For the [FSI] treated samples, the results were consistent with the removal of RTIL during the etching process. Small quantities of nitrides at ca. $398 \mathrm{eV}$ were also identified on the etched surfaces.

The $\mathrm{S} 2 \mathrm{p}$ high-resolution spectra displayed peaks corresponding to $-\mathrm{SO}_{2} \mathrm{CF}_{3}$ species for the [TFSI] treated samples at ca. 169 and $170.2 \mathrm{eV}$ (doublet) and $-\mathrm{SO}_{2} \mathrm{~F}$ for the [FSI] treated samples at ca. $169.7 \mathrm{eV}$ and 
$170.9 \mathrm{eV}$, oxidised sulphur species from 167.5 to $169.0 \mathrm{eV}$ and a range of peaks detected only in the etched samples and associated with sulphide species ranging from 163 to $165 \mathrm{eV}$. The overall quantity of sulphur-based species remained the same before and after etching.

A clear difference is visible between the Al surfaces treated in the neat ILs and in the hybrid solutions. The results suggest that the film formed on Al foil was thicker in the hybrid solutions with significant amount of reduced species associated with the IL anion. The film formed on Al foil was composed of an outer layer of Al oxide. The etching process indicated that $\mathrm{AlF}_{3}$ species dominate the film further in. The cation structure does not seem to be affected by the anodising process, according to the $\mathrm{N} 1 \mathrm{~s}$ region spectra, and this is in agreement with FTIR analysis described previously.

\section{DISCUSSION}

It has been reported in the literature that the aluminium substrate dissolves as $\mathrm{Al}^{3+}$ ions when subjected to higher oxidation potentials. ${ }^{13}$ The dissolved aluminium ions react with the electrolyte mixture to form aluminium salts, the nature of which depends on the salt and solvents in the electrolyte system. It has been shown previously that the [FSI] $]^{-}$anion is corrosive towards the aluminium substrate upon anodic polarisation $(>4.0 \mathrm{~V} \mathrm{vs.} \mathrm{Li} / \mathrm{Li}$ $+{ }^{29}$ It was also reported that the extent of corrosion was suppressed in LiTFSI electrolytes by the addition of $\mathrm{LiPF}_{6}$ salt, which resulted in passivation of the Al substrate. ${ }^{14,52}$ The layer that was formed on the Al substrate was found to be composed mainly of $\mathrm{AlF}_{3}$, as a result of the chemical attack of $\mathrm{LiPF}_{6}$ on the native oxide layer $\left(\mathrm{Al}_{2} \mathrm{O}_{3}\right)$.

In this work, the electrochemical studies indicate the passivation properties of the hybrid electrolytes in comparison to the conventional electrolyte. Poor passivation was observed for the aluminium substrate that was cycled by using the conventional (LP40) electrolyte. The anodised Al foil displayed signs of corrosion which was identified from the electrochemical experiments and was in agreement with SEM images. In the case of the hybrid electrolytes, the decreasing current density and flat CV profiles observed in the second cycle measurements indicate the effectiveness of the passivation on the Al surface. By correlating depth-profiling AI 2p XPS spectra of the aluminium samples with SEM images further clarification of the corrosion-inhibiting properties of the hybrid electrolytes can be determined. $\mathrm{Al}_{2} \mathrm{O}_{3}$ is the major Al species on the $\mathrm{Al}$ foil that was anodised in the neat ILs (without LP40). In contrast, $\mathrm{AlF}_{3}$ dominates over $\mathrm{Al}_{2} \mathrm{O}_{3}$ when the $\mathrm{Al}$ foil is anodised in the hybrid electrolytes that contain LP40. It is believed that the composite passive film that is formed with the help of the ionic liquid and the $\mathrm{PF}_{6}$ anion from the lithium salt in the electrolyte helps in the formation of a more robust passive film, which remains stable over the cycles performed here (confirmed by CV and chronoamperometry measurements). In particular, the [TFSI]-based hybrid electrolyte (HE 1) allowed the formation of a film that was mainly composed of $\mathrm{AlF}_{3}$. This suggests the formation of a passivating layer more resistant to electrochemical process. This was also observed via optical, microscopic and spectroscopic analyses, which indicated the predominant involvement of the anion chemistry in surface film formation. The pictorial representation of the composition of the passive film is presented in Figure S12.

Although [TFSI]-based lithium salts are known for their corrosive behavior on aluminium substrates, ${ }^{13,52,53}$ such behaviour was not observed when the [TFSI] anion was associated with the ionic liquid. ${ }^{17,54,55}$ The present work also confirms the corrosion inhibition and better passivation of the [TFSI]-based hybrid electrolyte (HE 1) in comparison to the [FSI]-based hybrid electrolyte ( $\mathrm{HE} 2$ ) and the conventional electrolyte. It is reported that the poor solubility of the passive film in the ionic liquid electrolytes helps prevent corrosion. ${ }^{54,55}$ Nevertheless, the better corrosion properties of HE 1 may also be due to the synergistic effect from both the ionic liquid and the $\mathrm{LiPF}_{6}{ }^{-}$salt being present in the electrolyte. In other words, the insoluble Al-TFSI-based species that form from the ionic liquid component and the $\mathrm{AlF}_{3}$ that forms predominantly (although not exclusively) from the $\mathrm{LiPF}_{6}$ salt component result in the suppression of the corrosion of the aluminium substrate in $\mathrm{HE} 1$.

This work has confirmed the significant corrosion inhibition properties of hybrid electrolytes based on a pyrrolidinium ionic liquid and commercial liquid electrolyte for lithium batteries. The hybrid electrolyte based on the bis(trifluoromethanesulfonyl)imide [TFSI] anion performs better than both the neat [TFSI] ionic liquid electrolyte and bis(fluorosulfonyl)imide [FSI] hybrid electrolyte in contact with aluminium at high anodic potentials $\left(4.7 \mathrm{~V} \mathrm{vs.} \mathrm{Li} / \mathrm{Li}^{+}\right)$. The organic carbonate electrolyte (neat LP40) did not exhibit appreciable passivation at such high anodic potentials. Cyclic voltammetry revealed that the neat [FSI] ionic liquid and hybrid electrolytes showed similar performance. However, differences were identified by potentiodynamic polarisation and effective passivation was only confirmed in the presence of $\mathrm{LiPF}_{6}$ in the hybrid electrolytes. Further differences were confirmed by microscopy and spectroscopy.

The observations that improved passivation occurred in the presence of the [TFSI] anion in the hybrid electrolyte and the correlation with an excess of $\mathrm{AlF}_{3}$ and anionic moieties in the surface film, strongly suggest that the IL anion may be critical to the anodic stability and high corrosion inhibition efficiency of aluminium current collectors. Further work is underway to investigate the influence of the chemistry of both the ionic liquid and organic solvent on speciation in a lithium cell configuration and correlate it with corrosion performance. This could lead to improved combinations to yield even better passivating properties of these hybrid electrolytes.

\section{METHODS}

Materials

Battery grade aluminium foils (MTI Corp.) were pre-cleaned in dimethyl carbonate solution (DMC, 99\%, Sigma-Aldrich) and dried in a vacuum oven at $120^{\circ} \mathrm{C}$. The dried aluminium foils were immediately transferred to the glove box. An electrolyte solution containing $1 \mathrm{M} \mathrm{LiPF}_{6}$ in ethylene carbonate and diethyl carbonate (LP40, Sigma-Aldrich, $<15$ ppm $\mathrm{H}_{2} \mathrm{O}$ and $<50 \mathrm{ppm} \mathrm{HF}$ ) was studied as the conventional electrolyte. Two hybrid electrolyte solutions containing $40 \mathrm{wt} \%$ of ionic liquid, $N$-methyl- $N$-propyl pyrrolidinium pyrrolidinium bis(trifluoromethanesulfonyl) imide, $\left[\mathrm{C}_{3} \mathrm{mpyr}\right]$ [TFSI] (Solvionic, France, 99.9\% purity) in LP40 (HE 1) as well as $N$-methyl- $N$ propyl pyrrolidinium bis(fluorosulfonyl)imide, $\left[\mathrm{C}_{3} \mathrm{mpyr}\right][\mathrm{FSI}]$ (Solvionic, France, 99.9\% purity) in LP40 (HE 2) were studied. In addition, the neat ionic liquids $\left[\mathrm{C}_{3} \mathrm{mpyr}\right]\left[\mathrm{TFSI}\right.$ and $\left[\mathrm{C}_{3} \mathrm{mpyr}\right][\mathrm{FSI}]$ were used for comparison studies.

Pretreatment of aluminium

The cleaned, dried aluminium foils were washed once more (inside the glove box) with dimethyl carbonate before the electrochemical measurements.

\section{Electrochemical measurements}

Electrochemical measurements were carried out in a pipette cell using a Biologic VMP3 multi-channel potentiostat, controlled by Biologic EC-Lab version 11.01 software. The pipette cell used was previously described elsewhere. ${ }^{24}$ The inner surface area of the pipette (equal to that of the working electrode W.E.) was $0.38 \pm 0.05 \mathrm{~cm}^{2}$. The cell consisted of a polyethylene pipette tip with an Ag/AgOTf reference and a Pt wire counter electrode of $0.5 \mathrm{~mm}$ diameter. Cyclic potentiodynamic polarisation (CPP) was carried out over the potential range $2.7-5 \mathrm{~V}$ vs. $\mathrm{Li}^{\mathrm{L}} \mathrm{Li}^{+}$at a scan rate of $10 \mathrm{mV} / \mathrm{min}$. The tafel curves were fitted using the EC lab software V10.44 (Bio Logic Science \& Instruments). Cyclic voltammetry (CV) measurements were carried out in the potential range of $2.7-4.7 \mathrm{~V} \mathrm{vs}$. $\mathrm{Li} / \mathrm{Li}^{+}$at a scan rate of $1 \mathrm{mV} / \mathrm{s}$ for three cycles followed by chronoamperometry measurements carried out for three hours at $4.7 \mathrm{~V}$ vs. $\mathrm{Li}^{\prime} / \mathrm{Li}^{+}$. Post-electrochemistry, the 
surfaces were rinsed with DMC to wash off any excess IL and finally dried under nitrogen stream. All electrochemical measurements were carried out inside a nitrogen glove box.

\section{Fourier-transform infrared spectroscopy (FTIR)}

Infrared spectra were acquired on a Perkin Elmer IR 101820 series spectrometer using the Spectrum (v.10.4) software. Ex situ attenuated total reflectance (ATR) spectra were obtained using a diamond ATR crystal in the mid-IR range $\left(4000-450 \mathrm{~cm}^{-1}\right)$. The rinsed and dried electrodes were hermetically transferred and loaded onto the sample stage after brief exposure to air. Firm pressure was applied to press the aluminium disk against the diamond window of the sample stage. All spectra were recorded with a $4 \mathrm{~cm}^{-1}$ resolution and 256 scans.

\section{Scanning electron microscopy (SEM) with energy dispersive x-ray spectroscopy (EDXS)}

Al surfaces after the electrochemical experiments were further characterised by SEM. SEM images of the Al electrodes were obtained with a JEOL JSM-IT300 at an accelerating voltage of $10 \mathrm{kV}$.

An Oxford Instruments X-Max $50 \mathrm{~mm}^{2}$ energy dispersive x-ray spectroscopy detector was used to analyse the Al surfaces, attachment at 10-11 mm W.D. with a collection time of five minutes. Oxford Instruments Aztec (version 1.0) software was used.

\section{Optical profilometry}

The aluminium coupons were examined using a Contour GT-K1 optical profilometer and the data were analysed with Vision 64 software v 5.30 , Bruker Instruments. The measurements were carried out with the following parameters: VSI $-20 \times$ or $50 \times$ lens, Magnifier $1.0 \times$, Speed $1 \times$, with a $25 \mu \mathrm{m}$ back scan, $50 \mu \mathrm{m}$ Length and $0.5 \%$ Threshold.

\section{X-ray photoelectron spectroscopy (XPS)}

For all XPS experiments, the binding energy scale was calibrated from the aliphatic hydrocarbon C $1 \mathrm{~s}$ peak at $284.8 \mathrm{eV}$. The samples were mounted on a XPS sample stud using conductive carbon adhesive tape and placed in a hermetic vessel for transfer to the instrument. XPS measurements were carried out at the Victorian node of the Australian National Fabrication Facility (CMSS, La Trobe University, Australia).

X-ray photoelectron spectroscopy was performed using an AXIS Ultra instrument (Kratos Analytical, Manchester, UK) utilising a monochromatic Al Ka x-ray source operated at $150 \mathrm{~W}(15 \mathrm{kV}, 10 \mathrm{~mA})$. The hybrid analysis mode used provided a sampling area of $300 \times 700 \mu \mathrm{m}^{2}$. Survey spectra were acquired at $80 \mathrm{eV}$ pass energy and high resolution region spectra were acquired at $20 \mathrm{eV}$ pass energy. Etching experiments were performed on the sample surface using an argon ion beam gun operating at $5 \mathrm{keV}$ and beam current of $2 \mu \mathrm{A}$, resulting in an estimated etching rate of $2 \AA . s^{-1}$. The pressure in the analysis chamber was maintained at $4.0 \times 10^{-10} \mathrm{mbar}$. Instrument operation was performed using the XPS software (Vision 2.0).

XPS data were analysed using the CASAXPS software (v. 2.3.16). Relative sensitivity factors taken from the Kratos Library were used to determine relative atomic percentages from survey and high-resolution scans of the most intense photoelectron peak for each element. Peak areas were measured after performing a two-point Shirley background subtraction. A Gaussian:Lorentzian algorithm (70:30\%) was used to fit the peaks to obtain quantitative results. The fit produces an estimated $\pm 10 \%$ error in the atomic concentration determined for each peak.

\section{Data availability}

The data sets generated during and/or analysed during the current study are available from the corresponding authors on reasonable request.

\section{ACKNOWLEDGEMENTS}

The authors would like to acknowledge the Victorian node of the Australian National Fabrication Facility (ANFF), a company established under the National Collaborative Research Infrastructure Strategy to provide nano and microfabrication facilities for researchers in Australia, through the La Trobe University Centre for Materials and Surface Science for the XPS facility. Funding from the Australia-India Strategic Research Fund (AISRF 48515) is gratefully acknowledged. DRM and MF acknowledge Australian Research Council support through their Laureate fellowship programs. S.M is thankful to the financial support provided by NCPRE (Grant No. 31/09/2015-16/
PVSE-R\&D) funded by Ministry of New Renewable Energy, Govt. of India. S.T is thankful to Reliance Industries, India and IITB Monash Research Academy for the scholarship and project grant.

\section{AUTHOR CONTRIBUTIONS}

The manuscript was written through the contribution of all authors. S.T. optimised and performed the electrochemical measurements and G.G. had carried out the surface analysis experiments. S.T. and G.G have contributed equally to the data analysis and manuscript preparation. P.H., M.F., S.M. and D.M.F have assisted in the project initiation, experimental planning, data analysis and reviewed the manuscript. All authors have given approval to the final version of the manuscript.

\section{ADDITIONAL INFORMATION}

Supplementary information accompanies the paper on the npj Materials Degradation website (https://doi.org/10.1038/s41529-018-0033-6).

Competing interests: The authors declare no competing interests.

Publisher's note: Springer Nature remains neutral with regard to jurisdictional claims in published maps and institutional affiliations.

\section{REFERENCES}

1. Zhang, Z. \& Zhang, S.S. (eds) Rechargeable Batteries (Springer International Publishing, 2015).

2. Huggins, R. A. Advanced Batteries Materials Science Aspects (Springer, 2009).

3. Whitehead, A. H. \& Schreiber, M. Current collectors for positive electrodes of lithium-based batteries. J. Electrochem. Soc. 152, A2105 (2005).

4. Braithwaite, J. W. et al. Corrosion of lithium-ion battery current collectors. J. Electrochem. Soc. 146, 448-456 (1999).

5. Evarts, E. C. Lithium batteries: to the limits of lithium. Nature 526, S93-S95 (2015).

6. Etacheri, V., Marom, R., Elazari, R., Salitra, G. \& Aurbach, D. Challenges in the development of advanced Li-ion batteries: a review. Energy Environ. Sci. 4, 3243-3262 (2011).

7. Yuge, R. et al. High energy density lithium ion batteries with iron- and nickelsubstituted lithium-rich layered oxide cathode. J. Electrochem. Soc. 163, A1881-A1885 (2016).

8. Choi, J. W. \& Aurbach, D. Promise and reality of post-lithium-ion batteries with high energy densities. Nat. Rev. Mater. 1, 16013 (2016).

9. Li, F., Gong, Y., Jia, G., Wang, Q. \& Peng, Z. A novel dual-salts of LiTFSI and LiODFB in LiFePO 4-based batteries for suppressing aluminum corrosion and improving cycling stability. J. Power Sources 295, 47-54 (2015).

10. Hyams, T. C., Go, J. \& Devine, T. M. Corrosion of aluminum current collectors in high-power lithium-ion batteries for use in hybrid electric vehicles. J. Electrochem. Soc. 154, C390 (2007).

11. Zhang, X., Winget, B., Doeff, M., Evans, J. W. \& Devine, T. M. Corrosion of aluminum current collectors in lithium-ion batteries with electrolytes containing LiPF6. J. Electrochem. Soc. 152, B448-B454 (2005).

12. Kramer, E. et al. Mechanism of anodic dissolution of the aluminum current collector in $1 \mathrm{M}$ LiTFSI EC:DEC 3:7 in rechargeable lithium batteries. J. Electrochem. Soc. 160, A356-A360 (2012).

13. $\mathrm{Xu}, \mathrm{K}$. Nonaqueous liquid electrolytes for lithium-based rechargeable batteries. Chem. Rev. 104, 4303-4418 (2004).

14. Morita, M., Shibata, T., Yoshimoto, N. \& Ishikawa, M. Anodic behavior of aluminum in organic solutions with different electrolytic salts for lithium ion batteries. Electrochim. Acta 47, 2787-2793 (2002).

15. Evans, T., Olson, J., Bhat, V. \& Lee, S. H. Effect of organic solvent addition to PYR13FSI+ LiFSI electrolytes on aluminum oxidation and rate performance of $\mathrm{Li}$ (Ni 1/3Mn1/3Co1/3)O2 cathodes. J. Power Sources 265, 132-139 (2014).

16. Kramer, E., Passerini, S. \& Winter, M. Dependency of aluminum collector corrosion in lithium ion batteries on the electrolyte solvent. ECS Electrochem. Lett. 1, C9-C11 (2012).

17. Moosbauer, D., Zugmann, S., Amereller, M. \& Gores, H. J. Effect of ionic liquids as additives on lithium electrolytes: conductivity, electrochemical stability, and aluminum corrosion. J. Chem. Eng. Data 55, 1794-1798 (2010).

18. Xu, K. Electrolytes and interphases in Li-lon batteries and Beyond. Chem. Rev. 114, 11503-11618 (2014).

19. Kühnel, R.-S., Lübke, M., Winter, M., Passerini, S. \& Balducci, A. Suppression of aluminum current collector corrosion in ionic liquid containing electrolytes. J. Power Sources 214, 178-184 (2012).

20. Theivaprakasam, S., MacFarlane, D. R. \& Mitra, S. Electrochemical studies of $N$ methyl $N$-propyl pyrrolidinium bis(trifluoromethanesulfonyl) imide ionic liquid 
mixtures with conventional electrolytes in LiFePO4/Li cells. Electrochim. Acta 180, 737-745 (2015)

21. Chen, R. et al. A facile approach of introducing DMS into LiODFB-PYR 14 TFS electrolyte for lithium-ion batteries. J. Mater. Chem. A 3, 6366-6372 (2015).

22. Peng, C., Yang, L., Wang, B., Zhang, Z. \& Li, N. Electrochemical behavior of aluminum foil in 1-alkyl-3-methylimidazolium tetrafluoroborate ionic liquids electrolytes. Chin. Sci. Bull. 51, 2824-2830 (2006).

23. Huang, P., Somers, A., Howlett, P. C. \& Forsyth, M. Film formation in trihexyl (tetradecyl) phosphonium diphenylphosphate ([P 6, 6, 6, 14][dpp]) ionic liquid on AA5083 aluminium alloy. Surf. Coat. Technol. 303, 385-395 (2016).

24. Chong, A. L., Mardel, J. I., MacFarlane, D. R., Forsyth, M. \& Somers, A. E. Synergistic corrosion inhibition of mild steel in aqueous chloride solutions by an imidazolinium carboxylate salt. ACS Sustain. Chem. Eng. 4, 1746-1755 (2016).

25. Somers, A. E., Chong, A. L., MacFarlane, D. R. \& Forsyth, M. Imidazolinium inhibitor for mild steel in aggressive conditions. in C\&P 2015: Corrosion and Prevention 2015. 1-8 (The Australasian Corrosion Association, Kerrimuir, Vic., 2015).

26. Latham, J.-A., Howlett, P. C., MacFarlane, D. R., Somers, A. \& Forsyth, M. Anodising AZ31 in a phosphonium ionic liquid: corrosion protection through composite film deposition. J. Electrochem. Soc. 159, C539-C545 (2012).

27. Cha, E.-H. et al. The corrosion study of al current collector in phosphonium ionic liquid as solvent for lithium ion battery. J. Korean Electrochem. Soc. 14, 152-156 (2011).

28. Allen, J. L. et al. N-Alkyl-N-methylpyrrolidinium difluoro(oxalato)borate ionic liquids: physical/electrochemical properties and Al corrosion. J. Power Sources 237, 104-111 (2013).

29. Cho, E. et al. Corrosion/passivation of aluminum current collector in bis(fluorosulfonyl)imide-based ionic liquid for lithium-ion batteries. Electrochem. Commun. 22, 1-3 (2012).

30. Yang, B., Li, C., Zhou, J., Liu, J. \& Zhang, Q. Pyrrolidinium-based ionic liquid electrolyte with organic additive and LiTFSI for high-safety lithium-ion batteries. Electrochim. Acta 148, 39-45 (2014).

31. Liu, C. et al. Ionic liquid electrolyte of lithium bis(fluorosulfonyl)imide/ $N$-methyl- $N$ propylpiperidinium bis(fluorosulfonyl)imide for Li/natural graphite cells: effect of concentration of lithium salt on the physicochemical and electrochemical properties. Electrochim. Acta 149, 370-385 (2014).

32. Navarra, M. A. lonic liquids as safe electrolyte components for Li-metal and Li-ion batteries. MRS Bull. 38, 548-553 (2013).

33. $\mathrm{Li}, \mathrm{H}$. et al. Application of a nonflammable electrolyte containing Pp13TFSI ionic liquid for lithium-ion batteries using the high capacity cathode material $\mathrm{Li}$ [Li0.2Mn0.54Ni0.13Co0.13]O2. RSC Adv. 3, 13907-13914 (2013).

34. Guerfi, A. et al. Improved electrolytes for Li-ion batteries: Mixtures of ionic liquid and organic electrolyte with enhanced safety and electrochemical performance. J. Power Sources 195, 845-852 (2010).

35. Choi, J. A., Shim, E. G., Scrosati, B. \& Kim, D. W. Mixed electrolytes of organic solvents and ionic liquid for rechargeable lithium-ion batteries. Bull. Korean Chem. Soc. 31, 3190-3194 (2010).

36. Matsuda, Y., Fukushima, T., Hashimoto, H. \& Arakawa, R. Solvation of lithium ions in mixed organic electrolyte solutions by electrospray ionization mass spectroscopy. J. Electrochem. Soc. 149, A1045-A1048 (2002).

37. Kühnel, R.-S., Böckenfeld, N., Passerini, S., Winter, M. \& Balducci, A. Mixtures of ionic liquid and organic carbonate as electrolyte with improved safety and performance for rechargeable lithium batteries. Electrochim. Acta 56, 4092-4099 (2011).

38. Yoon, H., Howlett, P. C., Best, A. S., Forsyth, M. \& MacFarlane, D. R. Fast charge/ discharge of Li metal batteries using an ionic liquid electrolyte. J. Electrochem. Soc. 160, A1629-A1637 (2013).

39. Zhou, Q., Henderson, W. A., Appetecchi, G. B., Montanino, M. \& Passerini, S. Physical and electrochemical properties of $N$-alkyl- $N$-methylpyrrolidinium bis (fluorosulfonyl)imide ionic liquids: PY ${ }_{13}$ FSI and PY ${ }_{14}$ FSI. J. Phys. Chem. B 112, 13577-13580 (2008).
40. Li, L. et al. Transport and electrochemical properties and spectral features of nonaqueous electrolytes containing LiFSI in linear carbonate solvents. J. Electrochem. Soc. 158, A74 (2011).

41. Howlett, P. C., Brack, N., Hollenkamp, A. F., Forsyth, M. \& MacFarlane, D. R. Characterization of the lithium surface in $\mathrm{N}$-methyl- $\mathrm{N}$-alkylpyrrolidinium bis (trifluoromethanesulfonyl) amide room-temperature ionic liquid electrolytes. J. Electrochem. Soc. 153, A595-A606 (2006).

42. Bednarska-Bolek, B., Jakubas, R., Bator, G. \& Baran, J. Vibrational study of the structural phase transition in bis(pyrrolidinium)-chloride-hexachloroantimonate (V) by infrared spectroscopy. J. Mol. Struct. 614, 151-157 (2002).

43. Yoon, H., Best, A. S., Forsyth, M., MacFarlane, D. R. \& Howlett, P. C. Physical properties of high Li-ion content $\mathrm{N}$-propyl-N-methylpyrrolidinium bis(fluorosulfonyl)imide based ionic liquid electrolytes. Phys. Chem. Chem. Phys. 17, 4656-4663 (2015).

44. Rey, I., Lassègues, J. C., Grondin, J. \& Servant, L. Infrared and Raman study of the PEO-LiTFSI polymer electrolyte. Electrochim. Acta 43, 1505-1510 (1998).

45. Colthup, N. B., Dalyse, H. L. \& Wiberley, S. E. Introduction to Infrared and Raman Spectroscopy (Academic Press, 1990)

46. Nyquist, C. L. P. R. A. \& Leugers, M. A. Handbook of Infrared and Raman Spectra of Inorganic Compounds and Organic Salts (Academic Press, 1997).

47. Forsyth, M. et al. New insights into the fundamental chemical nature of ionic liquid film formation on magnesium alloy surfaces. ACS Appl. Mater. Interfaces 1, 1045-1052 (2009).

48. Wagner, C. D. \& Muilenberg, G. E. Handbook of X-ray Photoelectron Spectroscopy: A Reference Book Of Standard Data For Use In X-ray Photoelectron Spectroscopy (Physical Electronics Division, Perkin-Elmer Corp., 1979).

49. Crist, B. A review of XPS data-banks. XPS Int. 1, 1-52 (2007).

50. Minami, I. lonic liquids in tribology. Molecules 14, 2286-2305 (2009).

51. Marcus, P., Hinnen, C., Imbert, D. \& Siffre, J. M. An in situ XPS study of the formation of aluminium-polymer interfaces. Surf. Interface Anal. 19, 127-132 (1992).

52. Krause, L. J. et al. Corrosion of aluminum at high voltages in non-aqueous electrolytes containing perfluoroalkylsulfonyl imides; new lithium salts for lithium-ion cells. J. Power Sources 68, 320-325 (1997).

53. Yang, H., Kwon, K., Devine, T. M. \& Evans, J. W. Aluminum corrosion in lithium batteries an investigation using the electrochemical quartz crystal microbalance aluminum corrosion in lithium batteries. J. Electrochem. Soc. 147, 4399-4407 (2000).

54. Kühnel, R.-S. \& Balducci, A. Comparison of the anodic behavior of aluminum current collectors in imide-based ionic liquids and consequences on the stability of high voltage supercapacitors. J. Power Sources 249, 163-171 (2014).

55. Kühnel, R.-S., Reiter, J., Jeong, S., Passerini, S. \& Balducci, A. Anodic stability of aluminum current collectors in an ionic liquid based on the (fluorosulfonyl)(trifluoromethanesulfonyl)imide anion and its implication on high voltage supercapacitors. Electrochem. Commun. 38, 117-119 (2014).

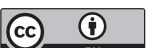

Open Access This article is licensed under a Creative Commons Attribution 4.0 International License, which permits use, sharing, adaptation, distribution and reproduction in any medium or format, as long as you give appropriate credit to the original author(s) and the source, provide a link to the Creative Commons license, and indicate if changes were made. The images or other third party material in this article are included in the article's Creative Commons license, unless indicated otherwise in a credit line to the material. If material is not included in the article's Creative Commons license and your intended use is not permitted by statutory regulation or exceeds the permitted use, you will need to obtain permission directly from the copyright holder. To view a copy of this license, visit http://creativecommons. org/licenses/by/4.0/.

(c) The Author(s) 2018 